\title{
A note on discontinuous Galerkin divergence-free solutions of the Navier-Stokes equations
}

\author{
Bernardo Cockburn * Guido Kanschat ${ }^{\dagger}$ \\ Dominik Schötzau $\ddagger$
}

\begin{abstract}
We present a class of discontinuous Galerkin methods for the incompressible Navier-Stokes equations yielding exactly divergence-free solutions. Exact incompressibility is achieved by using divergence-conforming velocity spaces for the approximation of the velocities. The resulting methods are locally conservative, energy-stable, and optimally convergent. We present a set of numerical tests that confirm these properties. The results of this note naturally expand the work in [15].
\end{abstract}

\section{Introduction}

In this note, we continue our study, [16], [13], [14] and [15], of discontinuous Galerkin (DG) methods for incompressible fluid flow and consider methods that provide an exactly divergence-free velocity approximation for the stationary incompressible Navier-Stokes equations

$$
\begin{aligned}
-\nu \Delta \boldsymbol{u}+\nabla \cdot(\boldsymbol{u} \otimes \boldsymbol{u})+\nabla p & =\boldsymbol{f} & & \text { in } \Omega, \\
\nabla \cdot \boldsymbol{u} & =0 & & \text { in } \Omega, \\
\boldsymbol{u} & =\mathbf{0} & & \text { on } \Gamma=\partial \Omega .
\end{aligned}
$$

As usual, $\nu$ is the kinematic viscosity, $\boldsymbol{u}$ the velocity, $p$ the pressure, and $\boldsymbol{f}$ the external body force. For the sake of simplicity, we take $\Omega$ to be a polygonal domain of $\mathbb{R}^{2}$.

Since this article is a follow-up of a whole series, let us put this work in perspective. In [16], DG methods for the Stokes equations were considered. Then in [13], these methods were extended to include a solenoidal convective

\footnotetext{
${ }^{*}$ School of Mathematics, University of Minnesota, 127 Vincent Hall, 206 Church St. SE, Minneapolis, MN 55455, USA, email: cockburn@math.umn.edu. Supported in part by NSF Grant DMS-0411254.

$\dagger$ Institut für Angewandte Mathematik, Universität Heidelberg, Im Neuenheimer Feld 293/294, 69120 Heidelberg, Germany, email: kanschat@dgfem.org.

$\ddagger$ Mathematics Department, University of British Columbia, 1984 Mathematics Road, Vancouver, BC V6T 1Z2, Canada, email: schoetzau@math.ubc.ca.
} 
velocity and the Oseen equations were considered; see also the review in [14]. Finally, in [15], DG methods for the Navier-Stokes equations were considered. This paper is devoted to expanding two aspects of the devising of those DG methods which were only cryptically addressed in [15].

To describe them, let us begin by pointing out that in [15], it was shown that DG methods for the incompressible Navier-Stokes equations cannot be both locally conservative as well as energy-stable unless the approximation to the convective velocity is exactly divergence-free. Then it was shown how to construct optimally convergent DG methods with these two properties; no other DG method has them. Such a construction was carried out by using totally discontinuous velocity spaces and the local discontinuous Galerkin (LDG) method to discretize the viscous terms. However, it was pointed out, see the second-last paragraph of the Introduction of the paper [15], that "exact incompressibility can be achieved trivially if the $L D G$ method has a velocity that is div-conforming, i.e., included in $H(\operatorname{div}, \Omega)$," and that the use of the LDG method for the discretization of the viscous terms was not really necessary since "any other $D G$ discretization whose primal form is both coercive and continuous could have been used." The purpose of this note is to elaborate on these two points.

The issue of providing exactly divergence-free approximations has been addressed in a few publications now. Indeed, exactly divergence-free approximations of the velocity have been obtained by Bastian and Rivière [6] in the framework of DG methods for Darcy's flow; this was achieved by means of a local post-processing similar to the one used in [15] for the Navier-Stokes equations. In [9], Carrero et al. obtained exactly divergence-free velocity approximations for a DG method that used spaces of solenoidal functions; the actual construction of those subspaces was avoided by using a hybridization technique. In [11] and [12], Cockburn and Gopalakrishnan used a similar technique for classical mixed methods using spaces of solenoidal functions. Finally, in [17] Cockburn et al. applied a technique similar to the one used in [15], to devise optimally convergent DG methods for incompressible elastic materials. The remarks developed in this note for the DG methods developed in [15] for the Navier-Stokes equations also hold for the DG methods developed in [17] for incompressible elastic materials.

The organization of the paper is as follows. In the following section, we review the LDG method for the Navier-Stokes equations in [15] with special attention to the use of $H(\operatorname{div} ; \Omega)$-conforming velocity spaces and its independence of the actual form of the DG scheme for the elliptic operator. In section 3, we show how the analytical results in [15] can be adjusted to these cases. Finally, the practical applicability of the schemes is demonstrated in section 4 .

\section{Description of the DG methods}

This section is devoted to the definition of locally conservative DG discretizations for the Navier-Stokes equations (1.1). We follow [15]. 


\subsection{Meshes and trace operators}

We denote by $\mathbb{T}_{h}$ a shape-regular triangulation of mesh-size $h$ of the domain $\Omega$ into triangles or quadrilaterals $\{K\}$. We further denote by $\mathcal{E}_{h}^{\mathcal{I}}$ the set of all interior edges of $\mathcal{T}_{h}$ and by $\mathcal{E}_{h}^{\mathcal{B}}$ the set of all boundary edges; we set $\mathcal{E}_{h}=\mathcal{E}_{h}^{\mathcal{I}} \cup \mathcal{E}_{h}^{\mathcal{B}}$.

Next, we introduce notation associated with traces: Let $K^{+}$and $K^{-}$be two adjacent elements of $\mathbb{T}_{h}$; let $\boldsymbol{x}$ be an arbitrary point of the interior edge $e=\partial K^{+} \cap \partial K^{-} \in \mathcal{E}_{h}^{\mathcal{I}}$. Let $\varphi$ be a piecewise smooth scalar-, vector-, or matrixvalued function and let us denote by $\varphi^{ \pm}$the traces of $\varphi$ on $e$ taken from within the interior of $K^{ \pm}$. Then, we define the mean value $\{\{\cdot\}$ at $\boldsymbol{x} \in e$ as

$$
\left\{\{\varphi\}:=\frac{1}{2}\left(\varphi^{+}+\varphi^{-}\right) .\right.
$$

Further, for a generic multiplication operator $\odot$, we define the jump $\llbracket \cdot \rrbracket$ at $\boldsymbol{x} \in e$ as

$$
\llbracket \varphi \odot \boldsymbol{n} \rrbracket:=\varphi^{+} \odot \boldsymbol{n}_{K^{+}}+\varphi^{-} \odot \boldsymbol{n}_{K^{-}} .
$$

Here, $\boldsymbol{n}_{K}$ denotes outward unit normal vector on the boundary $\partial K$ of element $K$. On boundary edges, we set accordingly $\{\varphi \varphi\}:=\varphi$, and $\llbracket \varphi \odot \boldsymbol{n} \rrbracket:=\varphi \odot \boldsymbol{n}$, with $\boldsymbol{n}$ denoting the outward unit normal vector on $\Gamma$.

\subsection{The DG methods for the Oseen equations}

We first introduce the DG methods for the Oseen equations. To do so, we write these equations in the form

$$
\begin{aligned}
\underline{\sigma} & =\nu \nabla u & & \text { in } \Omega, \\
-\nabla \cdot \underline{\sigma}+(\boldsymbol{w} \cdot \nabla) \boldsymbol{u}+\nabla p & =\boldsymbol{f} & & \text { in } \Omega, \\
\nabla \cdot \boldsymbol{u} & =0 & & \text { in } \Omega, \\
\boldsymbol{u} & =\mathbf{0} & & \text { on } \Gamma .
\end{aligned}
$$

We seek DG approximations to $(\underline{\sigma}, \boldsymbol{u}, p)$ in the space $\underline{\Sigma}_{h} \times \boldsymbol{V}_{h} \times Q_{h}$ where

$$
\begin{aligned}
\underline{\Sigma}_{h} & =\left\{\underline{\tau} \in L^{2}(\Omega)^{2 \times 2}:\left.\underline{\tau}\right|_{K} \in \underline{S}(K)=\boldsymbol{S}(K)^{2}, K \in \mathbb{T}_{h}\right\}, \\
\boldsymbol{V}_{h} & =\left\{\boldsymbol{v} \in H(\operatorname{div} ; \Omega):\left.\boldsymbol{v}\right|_{K} \in \boldsymbol{V}(K), K \in \mathbb{T}_{h} ; \boldsymbol{v} \cdot \boldsymbol{n}=0 \text { on } \Gamma\right\}, \\
Q_{h} & =\left\{q \in L^{2}(\Omega):\left.q\right|_{K} \in Q(K), K \in \mathbb{T}_{h} ; \int_{\Omega} q d \boldsymbol{x}=0\right\},
\end{aligned}
$$

where the local spaces $\underline{S}(K) \times \boldsymbol{V}(K) \times Q(K)$ satisfy the mild conditions

$$
\begin{aligned}
\nabla \boldsymbol{V}(K) & \subseteq \underline{S}(K), \\
\nabla \cdot \boldsymbol{V}(K) & \subseteq Q(K) .
\end{aligned}
$$

The first condition serves to ensure the existence and uniqueness of the approximation (see $[10,2]$ ). We will see later that the second guarantees that the approximate velocity is exactly incompressible provided that it is only weakly incompressible. Additionally, we require an inf-sup condition for the spaces $\boldsymbol{V}_{h}$ and $Q_{h}$. 
We define the approximate solution $\left(\underline{\sigma}_{h}, \boldsymbol{u}_{h}, p_{h}\right) \in \underline{\Sigma}_{h} \times \boldsymbol{V}_{h} \times Q_{h}$ by requesting that

$$
\begin{aligned}
& \sum_{K \in \mathbb{T}_{h}} \int_{K} \underline{\sigma}_{h}: \underline{\tau} d \boldsymbol{x}=\sum_{K \in \mathbb{T}_{h}}\left(-\nu \int_{K} \boldsymbol{u}_{h} \cdot \nabla \cdot \underline{\tau} d \boldsymbol{x}+\nu \int_{\partial K} \widehat{\boldsymbol{u}}_{h}^{\sigma} \cdot \underline{\tau} \cdot \boldsymbol{n}_{K} d s\right), \\
& \sum_{K \in \mathbb{T}_{h}}\left(\int_{K} \underline{\sigma}_{h}: \nabla \boldsymbol{v} d \boldsymbol{x}-\int_{\partial K} \underline{\underline{\sigma}}_{h}:\left(\boldsymbol{v} \otimes \boldsymbol{n}_{K}\right) d s-\int_{K} p_{h} \nabla \cdot \boldsymbol{v} d \boldsymbol{x}\right) \\
& +\sum_{K \in \mathbb{T}_{h}}\left(-\int_{K} \boldsymbol{u}_{h} \cdot \nabla \cdot(\boldsymbol{v} \otimes \boldsymbol{w}) d \boldsymbol{x}+\int_{\partial K} \boldsymbol{w} \cdot \boldsymbol{n}_{K} \widehat{\boldsymbol{u}}_{h}^{\boldsymbol{w}} \cdot \boldsymbol{v} d s\right)=\int_{\Omega} \boldsymbol{f} \cdot \boldsymbol{v} d \boldsymbol{x}, \\
& -\sum_{K \in \mathbb{T}_{h}}\left(\int_{K} \boldsymbol{u}_{h} \cdot \nabla q d \boldsymbol{x}+\int_{\partial K} \widehat{\boldsymbol{u}}_{h}^{p} \cdot \boldsymbol{n}_{K} q d s\right)=0,
\end{aligned}
$$

for all test functions $(\underline{\tau}, \boldsymbol{v}, q) \in \underline{\Sigma}_{h} \times \boldsymbol{V}_{h} \times Q_{h}$. Here $\widehat{\boldsymbol{u}}_{h}^{\sigma}, \widehat{\underline{\sigma}}_{h}, \widehat{\boldsymbol{u}}_{h}^{\boldsymbol{w}}$ and $\widehat{\boldsymbol{u}}_{h}^{p}$ are the numerical fluxes that are defined as follows.

\section{The convective numerical trace}

For the convective numerical trace $\widehat{\boldsymbol{u}}_{h}^{\boldsymbol{w}}$ in (2.4b), we proceed exactly as in [15] and take

$$
\widehat{\boldsymbol{u}}_{h}^{\boldsymbol{w}}(\boldsymbol{x})= \begin{cases}\lim _{\varepsilon \downarrow 0} \boldsymbol{u}_{h}(\boldsymbol{x}-\varepsilon \boldsymbol{w}(\boldsymbol{x})), & \boldsymbol{x} \in \partial K \backslash \Gamma_{-}, \\ \mathbf{0}, & \boldsymbol{x} \in \partial K \cap \Gamma_{-},\end{cases}
$$

where $\Gamma_{-}^{\boldsymbol{w}}=\{\boldsymbol{x} \in \Gamma: \boldsymbol{w}(\boldsymbol{x}) \cdot \boldsymbol{n}(\boldsymbol{x})<0\}$.

\section{The numerical traces related to the incompressibility constraint}

We first notice that, since the space of velocities $\boldsymbol{V}_{h}$ is included in $H(\operatorname{div} ; \Omega)$, the jump of the normal component of $\boldsymbol{v}$ vanishes over edges. Therefore, unlike for the case treated in [15], there is no need to introduce a numerical flux associated with $p_{h}$.

We take the flux $\widehat{u}_{h}^{p}$ as in [15], that is,

$$
\widehat{\boldsymbol{u}}_{h}^{p}=\left\{\left\{\boldsymbol{u}_{h}\right\}\right\} \text { on } \mathcal{E}_{h}^{\mathcal{I}} \quad \text { and } \quad \widehat{\boldsymbol{u}}_{h}^{p}=\mathbf{0} \text { on } \Gamma .
$$

notice that with this choice, since $\boldsymbol{V}_{h} \subset H($ div; $\Omega$ ), we obtain, by integration by parts, that

$$
\sum_{K \in \mathbb{T}_{h}}\left(-\int_{K} \boldsymbol{u}_{h} \cdot \nabla q d \boldsymbol{x}+\int_{\partial K} \widehat{\boldsymbol{u}}_{h}^{p} \cdot \boldsymbol{n}_{K} q d s\right)=\int_{\Omega} q \nabla \cdot \boldsymbol{u}_{h} d \boldsymbol{x} .
$$

Moreover, due to the boundary condition in $\boldsymbol{V}_{h}$ and the inclusion in (2.3b), we have that

$$
\int_{\Omega} \nabla \cdot \boldsymbol{u}_{h} d \boldsymbol{x}=0 \quad \text { and } \quad \nabla \cdot \boldsymbol{u}_{h} \in Q_{h}
$$


We can immediately see from this identity and from equation $(2.4 \mathrm{c})$ that, if condition $(2.3 \mathrm{~b})$ is satisfied, the approximate velocity $\boldsymbol{u}_{h}$ is automatically exactly divergence-free. Let us point out that this condition does not follow if the boundary condition $\boldsymbol{v} \cdot \boldsymbol{n}=0$ on $\Gamma$ is not incorporated in the velocity space $\boldsymbol{V}_{h}$ given by $(2.2 \mathrm{~b})$.

\section{The diffusive numerical traces}

Notice that, since the diffusion equation

$$
-\nu \Delta \boldsymbol{u}=\boldsymbol{g}
$$

can be rewritten as

$$
\boldsymbol{\sigma}_{i}=\nu \nabla u_{i} \quad \text { and } \quad-\nabla \cdot \boldsymbol{\sigma}_{i}=g_{i}
$$

for $i=1,2$, we see that the discretization of the vector equation can be done by discretizing similar scalar equations. So, the definition of the the numerical traces $\underline{\widehat{\sigma}}_{h}$ and $\widehat{\boldsymbol{u}}_{h}^{\sigma}$ can be obtained in a component-by-component basis. In Table 2.1 , taken (and slightly modified) from [2], we can see the main choices of those numerical traces that can be found in the literature for the scalar equation

$$
\boldsymbol{\sigma}=\nu \nabla u \quad \text { and } \quad-\nabla \cdot \boldsymbol{\sigma}=g .
$$

There, we restrict ourselves to adjoint-consistent methods. The parameter

Table 2.1: Some DG methods and their numerical fluxes for the scalar equation (2.7).

\begin{tabular}{lcc}
\hline Method & $\widehat{u}_{h}$ & $\widehat{\boldsymbol{\sigma}}_{h}$ \\
\hline Bassi-Rebay [4] & $\left\{u_{h}\right\}$ & $\left\{\boldsymbol{\sigma}_{h}\right\}$ \\
Brezzi et al. [8] & $\left\{u_{h}\right\}$ & $\left\{\boldsymbol{\sigma}_{h}\right\}-\boldsymbol{\alpha}_{\mathrm{r}}\left(\llbracket u_{h} \boldsymbol{n} \rrbracket\right)$ \\
LDG [18] & $\left\{u_{h}\right\}-\boldsymbol{\beta} \cdot \llbracket u_{h} \boldsymbol{n} \rrbracket$ & $\left\{\boldsymbol{\sigma}_{h}\right\}+\boldsymbol{\beta} \llbracket \sigma_{h} \cdot \boldsymbol{n} \rrbracket-\boldsymbol{\alpha}_{\mathrm{j}}\left(\llbracket u_{h} \boldsymbol{n} \rrbracket\right)$ \\
IP [1] & $\left\{u_{h}\right\}$ & $\left\{\nabla_{h} u_{h}\right\}-\boldsymbol{\alpha}_{\mathrm{j}}\left(\llbracket u_{h} \boldsymbol{n} \rrbracket\right)$ \\
IP with $\left|\boldsymbol{\beta} \cdot \boldsymbol{n}_{K}\right|=\frac{1}{2}[3]$ & $\left\{u_{h}\right\}-\boldsymbol{\beta} \cdot \llbracket u_{h} \boldsymbol{n} \rrbracket$ & $\left.\left\{\nabla_{h} u_{h}\right\}+\boldsymbol{\beta} \llbracket \sigma_{h} \cdot \boldsymbol{n} \rrbracket-\boldsymbol{\alpha}_{\mathrm{j}} \llbracket u_{h} \boldsymbol{n} \rrbracket\right)$ \\
Bassi et al. [5] & $\left\{u_{h}\right\}$ & $\left\{\nabla_{h} u_{h}\right\}-\boldsymbol{\alpha}_{\mathrm{r}}\left(\llbracket u_{h} \boldsymbol{n} \rrbracket\right)$ \\
\hline
\end{tabular}

$\boldsymbol{\beta}$ can be incorporated in the fluxes of the LDG and IP methods in order to increase their accuracy. The piecewise gradient is denoted by $\nabla_{h}$. On a face $e$ the stabilization functions $\boldsymbol{\alpha}_{\mathrm{j}}$ and $\boldsymbol{\alpha}_{\mathrm{r}}$ are defined by

$$
\boldsymbol{\alpha}_{\mathrm{j}}(\phi)=\eta_{e} h_{e}^{-1} \boldsymbol{\phi} \quad \text { and } \quad \boldsymbol{\alpha}_{\mathrm{r}}(\phi)=-\eta_{e}\left\{\left\{\boldsymbol{r}_{e}(\phi)\right\},\right.
$$


where $h_{e}$ is the orthogonal length of the cells adjacent to the edge under consideration, $\eta_{e}$ is a parameter that has to be chosen independently of the mesh size, and $\boldsymbol{r}_{e}(\boldsymbol{\phi}) \in \boldsymbol{\Sigma}_{\boldsymbol{h}}$ is given by

$$
\int_{\Omega} \boldsymbol{r}_{e}(\boldsymbol{\phi}) \cdot \boldsymbol{\tau} d \boldsymbol{x}=-\int_{e} \boldsymbol{\phi} \cdot\left\{\{\boldsymbol{\tau}\} d s \quad \forall \boldsymbol{\tau} \in \boldsymbol{\Sigma}_{h}\right.
$$

The vector-valued space $\boldsymbol{\Sigma}_{h}$ is given by

$$
\boldsymbol{\Sigma}_{h}=\left\{\boldsymbol{\tau} \in L^{2}(\Omega)^{2}:\left.\boldsymbol{\tau}\right|_{K} \in \boldsymbol{S}(K), K \in \mathbb{T}_{h}\right\},
$$

where $\boldsymbol{S}(K)$ is a the vector-valued component of $\underline{S}(K)=\boldsymbol{S}(K)^{2}$.

This completes the definition of the DG methods for the Oseen problem (2.1).

\subsection{Compact formulation of the DG methods}

We now present the compact formulation of the DG methods obtained by eliminating the auxiliary variable $\underline{\sigma}_{h}$. Indeed, by doing so, the approximation $\left(\boldsymbol{u}_{h}, p_{h}\right) \in \boldsymbol{V}_{h} \times Q_{h}$ of the Oseen problem satisfies

$$
\begin{aligned}
A_{h}\left(\boldsymbol{u}_{h}, \boldsymbol{v}\right)+O_{h}\left(\boldsymbol{w} ; \boldsymbol{u}_{h}, \boldsymbol{v}\right)+B_{h}\left(\boldsymbol{v}, p_{h}\right) & =\int_{\Omega} \boldsymbol{f} \cdot \boldsymbol{v} d \boldsymbol{x}, \\
-B_{h}\left(\boldsymbol{u}_{h}, q\right) & =0,
\end{aligned}
$$

for all $(\boldsymbol{v}, q) \in \boldsymbol{V}_{h} \times Q_{h}$. Here, the forms $A_{h}, O_{h}$ and $B_{h}$ are associated to the above DG discretizations of the convective terms, the Laplacian, and the incompressibility. The convective form is given by

$$
O_{h}(\boldsymbol{w} ; \boldsymbol{u}, \boldsymbol{v}):=-\sum_{K \in \mathbb{T}_{h}} \int_{K} \boldsymbol{u} \cdot \nabla \cdot(\boldsymbol{v} \otimes \boldsymbol{w}) d \boldsymbol{x}+\sum_{K \in \mathbb{T}_{h}} \int_{\partial K \backslash \Gamma_{-}^{\boldsymbol{w}}} \boldsymbol{w} \cdot \boldsymbol{n}_{K} \widehat{\boldsymbol{u}}^{\boldsymbol{w}} \cdot \boldsymbol{v} d s .
$$

The bilinear form $A_{h}(\boldsymbol{u}, \boldsymbol{v})$ can take many forms according to the choice of numerical traces we take, cf. Table 2.1. For example, if we choose the LDG method with $\boldsymbol{\beta}=\mathbf{0}$, we have

$$
\begin{aligned}
A_{h}(\boldsymbol{u}, \boldsymbol{v})=\int_{\Omega} \nu\left[\nabla_{h} \boldsymbol{u}-\underline{\mathcal{L}}(\boldsymbol{u})\right]: & {\left[\nabla_{h} \boldsymbol{v}-\underline{\mathcal{L}}(\boldsymbol{v})\right] d \boldsymbol{x} } \\
+ & \sum_{e \in \mathbb{E}_{h}} \int_{e} \kappa \llbracket \boldsymbol{u} \otimes \boldsymbol{n} \rrbracket: \llbracket \boldsymbol{v} \otimes \boldsymbol{n} \rrbracket d s,
\end{aligned}
$$

where $\kappa>0$ is a stabilization function, and the lifting operator $\underline{\mathcal{L}}: \boldsymbol{V}_{h} \rightarrow \underline{\Sigma}_{h}$ is given by

$$
\int_{\Omega} \underline{\mathcal{L}}(\boldsymbol{v}): \underline{\tau} d \boldsymbol{x}=\sum_{e \in \mathbb{E}_{h}} \int_{e} \llbracket \boldsymbol{v} \otimes \boldsymbol{n} \rrbracket:\{\{\underline{\tau}\}] d s \quad \forall \underline{\tau} \in \underline{\Sigma}_{h}
$$

This is the choice we used in [15]. Notice that we have used the condition (2.3a). 
If we choose instead the (symmetric) interior penalty (IP) [1], the bilinear form $A_{h}$ for the vector valued Laplacian is given by

$$
\begin{aligned}
& A_{h}(\boldsymbol{u}, \boldsymbol{v})=\int_{\Omega} \nu \nabla_{h} \boldsymbol{u}: \nabla_{h} \boldsymbol{v} d \boldsymbol{x}-\sum_{e \in \mathbb{E}_{h}} \int_{e} \llbracket \boldsymbol{v} \otimes \boldsymbol{n} \rrbracket:\left\{\left\{\nu \nabla_{h} u\right\} d s\right. \\
& \quad-\sum_{e \in \mathbb{E}_{h}} \int_{e} \llbracket \boldsymbol{u} \otimes \boldsymbol{n} \rrbracket:\left\{\left\{\nu \nabla_{h} \boldsymbol{v}\right\}\right\} d s+\sum_{e \in \mathbb{E}_{h}} \int_{e} \kappa \llbracket \boldsymbol{u} \otimes \boldsymbol{n} \rrbracket: \llbracket \boldsymbol{v} \otimes \boldsymbol{n} \rrbracket d s,
\end{aligned}
$$

for $\boldsymbol{u}, \boldsymbol{v} \in \boldsymbol{V}_{h}$. Again, notice that we used condition (2.3a). The penalty function $\kappa$ is chosen so as to ensure that the form $A_{h}$ is positive definite. It is this form that we will present numerical results for in section 4 .

Finally, the form $B_{h}$ is given by

$$
B_{h}(\boldsymbol{v}, q)=-\int_{\Omega} \nabla \cdot \boldsymbol{v} q d \boldsymbol{x} .
$$

\subsection{The DG methods for the Navier-Stokes equations}

Using the compact formulation that we introduced in for the Oseen problem, we consider the following DG methods for the Navier-Stokes equations: find $\left(\boldsymbol{u}_{h}, p_{h}\right) \in \boldsymbol{V}_{h} \times Q_{h}$ such that

$$
\begin{aligned}
A_{h}\left(\boldsymbol{u}_{h}, \boldsymbol{v}\right)+O_{h}\left(\boldsymbol{w} ; \boldsymbol{u}_{h}, \boldsymbol{v}\right)+B_{h}\left(\boldsymbol{v}, p_{h}\right) & =\int_{\Omega} \boldsymbol{f} \cdot \boldsymbol{v} d \boldsymbol{x}, \\
-B_{h}\left(\boldsymbol{u}_{h}, q\right) & =0,
\end{aligned}
$$

and

$$
\boldsymbol{w}=\boldsymbol{u}_{h},
$$

for all $(\boldsymbol{v}, q) \in \boldsymbol{V}_{h} \times Q_{h}$. The unusual separation of the variables $\boldsymbol{u}_{h}$ and $w$ in the convective term of $(2.12 \mathrm{a})$ was introduced in [15] in order to introduce a postprocessing instead of the equality (2.12c). Here, we keep it in order to stress the relation to the Oseen system. Since $\boldsymbol{w}=\boldsymbol{u}_{h}$ is exactly divergence-free, the resulting DG methods are locally conservative and energy-stable; cf. [15].

The approximation $\left(\boldsymbol{u}_{h}, p_{h}\right)$ can be found by employing the classical Picard iteration: given $\left(\boldsymbol{u}_{h}^{k-1}, p_{h}^{k-1}\right)$, find the new iterate $\left(\boldsymbol{u}_{h}^{k}, p_{h}^{k}\right)$ that satisfies the Oseen problem

$$
\begin{aligned}
A_{h}\left(\boldsymbol{u}_{h}^{k}, \boldsymbol{v}\right)+O_{h}\left(\boldsymbol{w} ; \boldsymbol{u}_{h}^{k}, \boldsymbol{v}\right)+B_{h}\left(\boldsymbol{v}, p_{h}^{k}\right) & =\int_{\Omega} \boldsymbol{f} \cdot \boldsymbol{v} d \boldsymbol{x} \\
-B_{h}\left(\boldsymbol{u}_{h}^{k}, q\right) & =0 \\
\boldsymbol{w} & =\boldsymbol{u}_{h}^{k-1}
\end{aligned}
$$

for all $(\boldsymbol{v}, q) \in \boldsymbol{V}_{h} \times Q_{h}$. Notice that all the velocity iterates $\boldsymbol{w}=\boldsymbol{u}_{h}^{k}$ are exactly divergence-free and therefore, we can employ the analysis in [13] to obtain solvability and approximation properties of each intermediate linear system.

As in [15] it can be shown that this iteration converges linearly for any initial approximation $\left(u_{h}^{0}, p_{h}^{0}\right)$, provided that $\nu^{-2}\|f\|_{0}$ is sufficiently small. 


\section{Analytical results}

In this section, we state and discuss the main properties of our discretization schemes. In order to do so, we first specify the pairs of spaces $\boldsymbol{V}_{h}$ and $Q_{h}$ we are going to use. Notice that we require spaces which fulfill the condition $(2.3 \mathrm{~b})$ on the pair of local spaces $\boldsymbol{V}(K) / Q(K)$. In [15], the Brezzi-Douglas-Marini element $B D M_{k+1} / \mathbb{P}_{k}$ pair was considered for simplicial and rectangular meshes. On simplicial meshes, the Raviart-Thomas pair $R T_{k} / \mathbb{P}_{k}$ could also be used since it also satisfies the condition (2.3b). In [21] the pair $\mathbb{Q}_{k+1}^{2} / \mathbb{Q}_{k}$ was used with rectangular element; however, since this pair does not satisfy (2.3b), the resulting velocity would not be divergence-free. We can use, instead, the Raviart-Thomas pair $R T_{k} / \mathbb{Q}_{k}$ since we do have that

$$
\nabla \cdot R T_{k}=\mathbb{Q}(K) .
$$

From now on, we restrict ourselves to working with this pair of local spaces. The resulting space $\boldsymbol{V}_{h}$ is equipped with the norm

$$
\|\boldsymbol{v}\|_{1, h}^{2}:=\sum_{K \in \mathbb{T}_{h}}\|\nabla \boldsymbol{v}\|_{0, K}^{2}+\sum_{e \in \mathbb{E}_{h}} \int_{e} h_{e}^{-1}|\llbracket \boldsymbol{v} \otimes \boldsymbol{n} \rrbracket|^{2} d s,
$$

Proposition 3.1 The parameter $\eta_{e}$ in (2.8) can be chosen such that the diffusive form $A_{h}\left(\boldsymbol{u}_{h}, \boldsymbol{v}\right)$ is coercive and bounded on $\boldsymbol{V}_{h}$. In other words, there exist constants $c_{1}$ and $c_{2}$ such that for all $\boldsymbol{u}, \boldsymbol{v} \in \boldsymbol{V}_{h}$ holds

$$
\begin{aligned}
& A_{h}\left(\boldsymbol{u}_{h}, \boldsymbol{v}\right) \leq \nu c_{1}\|\boldsymbol{u}\|_{1, h}\|\boldsymbol{v}\|_{1, h}, \\
& A_{h}\left(\boldsymbol{u}_{h}, \boldsymbol{u}\right) \geq \nu c_{2}\|\boldsymbol{u}\|_{1, h}^{2} .
\end{aligned}
$$

Proof: This proposition is a direct consequence of the analysis in [2] if we consider that $\boldsymbol{V}_{h}$ is a subspace of the vector valued DG spaces based on $\mathbb{Q}_{k+1}$.

Proposition 3.2 The advective form $O_{h}\left(\boldsymbol{w} ; \boldsymbol{u}_{h}, \boldsymbol{v}\right)$ has a nonnegative symmetric part, that is for any $\boldsymbol{w}, \boldsymbol{u} \in \boldsymbol{V}_{h}$ holds

$$
O_{h}(\boldsymbol{w} ; \boldsymbol{u}, \boldsymbol{u}) \geq 0 .
$$

Furthermore, it is Lipschitz continuous in its first argument,

$$
\left|O_{h}\left(\boldsymbol{w}_{\mathbf{1}} ; \boldsymbol{u}, \boldsymbol{v}\right)-O_{h}\left(\boldsymbol{w}_{\mathbf{2}} ; \boldsymbol{u}, \boldsymbol{v}\right)\right| \leq C_{o}\left\|\boldsymbol{w}_{\mathbf{1}}-\boldsymbol{w}_{\mathbf{2}}\right\|_{1, h}\|\boldsymbol{u}\|_{1, h}\|\boldsymbol{v}\|_{1, h}
$$

Proof: Since $\boldsymbol{V}_{h} \subset H(\operatorname{div} ; \Omega)$, we can immediately apply the proof of the corresponding propositions 4.2 and 4.3 in [15].

Proposition 3.3 The form $B_{h}$ is continuous and

$$
\left|B_{h}(\boldsymbol{v}, q)\right| \leq \sqrt{2}\|v\|_{1, h}\|q\|_{0} \quad \forall(\boldsymbol{v}, q) \in \boldsymbol{V}(h) \times L^{2}(\Omega) .
$$

Furthermore, the velocity-pressure pair $\boldsymbol{V}_{h} \times Q_{h}$ is inf-sup stable and satisfies

$$
\inf _{q \in Q_{h}} \sup _{\boldsymbol{v} \in \boldsymbol{V}_{h}} \frac{B_{h}(\boldsymbol{v}, q)}{\|\boldsymbol{v}\|_{1, h}\|q\|_{0}} \geq \beta>0,
$$

for a constant $\beta$ independent of the mesh size. 
Proof: This proposition was proven in [21, Theorem 6.12].

With these propositions, we derive the following result:

Theorem 3.1 Under the above assumptions, Theorem 4.7 (on the existence and uniqueness of the approximate solution) and Theorem 4.8 (a priori error estimates) in [15] hold for the DG methods defined in Section 2. In particular, we have the optimal error bound

$$
\left\|\boldsymbol{u}-\boldsymbol{u}_{h}\right\|_{1, h}+\left\|p-p_{h}\right\|_{0} \leq C h^{k}\left(\|\boldsymbol{u}\|_{k+1}+\|p\|_{k}\right),
$$

with a constant $C$ independent of the mesh size. Moreover, the approximate velocity $\boldsymbol{u}_{h}$ is exactly divergence-free.

The proof of the result in Theorem 3.1 follows then almost word by word those of the above mentioned results in [15].

We remark that this result is optimal for the error of the velocity, since the space $R T_{k}$ contains $\mathbb{P}_{k}$ as largest complete polynomial space; therefore, the additional functions in $R T_{k}$ contribute to the stability of the method and to the construction of a solenoidal solution, but not to the asymptotic quality of the error estimate.

\section{Numerical Examples}

To carry out our numerical experiments, we take the symmetric interior penalty method. We consider rectangular mesh cells and the local spaces are, since this method does not use a separate space for $\underline{\sigma}$,

$$
\boldsymbol{V}(K) \times Q(K)=R T_{k}(K) \times \mathbb{Q}_{k}(K),
$$

where $\mathbb{Q}_{k}$ is the space of tensor product polynomials of degree $k$ and $R T_{k}(K)$ is the Raviart-Thomas space of degree $k$. This space is constructed from anisotropic polynomials in such a way that the vector component $u_{i}$ is a product of polynomials

$$
u_{i}\left(x_{1}, \ldots, x_{d}\right)=\prod_{j=1}^{d} p_{j}^{i}\left(x_{j}\right)
$$

with $p_{j}^{i} \in \mathbb{P}_{k+1}(K)$ if $j=i$ and else in $\mathbb{P}_{k}(K)$.

Since this function has boundary values different from zero, special precautions must be taken. As pointed out in Section 2, in order to obtain a divergencefree solution in the elements adjacent to the boundary, the Dirichlet boundary condition for the normal component must be implemented in a strong way, while the tangential components obtain their boundary values weakly through the form (2.11). The strong boundary values are obtained by interpolating the normal component of the prescribed boundary function in the set of Gauss points required to integrate $\mathrm{RT}_{k}$ exactly on a face and then initializing the start 


\begin{tabular}{|c|c|cc|cc|cc|c|}
\hline$k$ & $L$ & $\left\|\nabla e_{u}\right\|$ & ord. & $\left\|e_{u}\right\|$ & ord. & $\|p\|$ & ord. & $\|$ div $u \|_{\infty}$ \\
\hline \multirow{4}{*}{1} & 4 & $9.7 \mathrm{e}+0$ & 1.01 & $2.3 \mathrm{e}-1$ & 1.82 & $3.9 \mathrm{e}+0$ & 1.63 & $8.7 \mathrm{e}-10$ \\
& 5 & $4.9 \mathrm{e}+0$ & 1.00 & $6.2 \mathrm{e}-2$ & 1.89 & $1.2 \mathrm{e}+0$ & 1.75 & $2.8 \mathrm{e}-09$ \\
& 6 & $2.4 \mathrm{e}+0$ & 1.00 & $1.6 \mathrm{e}-2$ & 1.93 & $3.3 \mathrm{e}-1$ & 1.81 & $6.7 \mathrm{e}-09$ \\
& 7 & $1.2 \mathrm{e}+0$ & 1.00 & $4.2 \mathrm{e}-3$ & 1.96 & $9.3 \mathrm{e}-2$ & 1.84 & $3.3 \mathrm{e}-08$ \\
& 8 & $6.1 \mathrm{e}-1$ & 1.00 & $1.1 \mathrm{e}-3$ & 1.98 & $2.6 \mathrm{e}-2$ & 1.86 & $2.8 \mathrm{e}-09$ \\
\hline \multirow{4}{*}{2} & 3 & $3.9 \mathrm{e}+0$ & 2.22 & $9.3 \mathrm{e}-2$ & 3.12 & $2.6 \mathrm{e}+0$ & 2.01 & $4.8 \mathrm{e}-10$ \\
& 4 & $9.9 \mathrm{e}-1$ & 1.97 & $1.2 \mathrm{e}-2$ & 2.96 & $4.5 \mathrm{e}-1$ & 2.53 & $1.7 \mathrm{e}-09$ \\
& 5 & $2.5 \mathrm{e}-1$ & 2.00 & $1.5 \mathrm{e}-3$ & 3.00 & $6.5 \mathrm{e}-2$ & 2.79 & $4.1 \mathrm{e}-09$ \\
& 6 & $6.2 \mathrm{e}-2$ & 2.00 & $1.9 \mathrm{e}-4$ & 3.00 & $9.2 \mathrm{e}-3$ & 2.82 & $2.0 \mathrm{e}-08$ \\
& 7 & $1.5 \mathrm{e}-2$ & 2.00 & $2.3 \mathrm{e}-5$ & 3.00 & $1.4 \mathrm{e}-3$ & 2.77 & $7.3 \mathrm{e}-08$ \\
\hline \multirow{4}{*}{3} & 4 & $6.5 \mathrm{e}-2$ & 2.99 & $6.8 \mathrm{e}-4$ & 3.78 & $4.2 \mathrm{e}-2$ & 3.46 & $1.9 \mathrm{e}-09$ \\
& 5 & $8.0 \mathrm{e}-3$ & 3.03 & $4.6 \mathrm{e}-5$ & 3.87 & $3.2 \mathrm{e}-3$ & 3.71 & $7.9 \mathrm{e}-09$ \\
& 6 & $9.8 \mathrm{e}-4$ & 3.02 & $3.0 \mathrm{e}-6$ & 3.93 & $2.5 \mathrm{e}-4$ & 3.72 & $2.0 \mathrm{e}-08$ \\
\hline \multirow{4}{*}{4} & 3 & $5.3 \mathrm{e}-2$ & 4.27 & $6.8 \mathrm{e}-4$ & 5.31 & $6.3 \mathrm{e}-2$ & 3.91 & $7.6 \mathrm{e}-10$ \\
& 4 & $3.4 \mathrm{e}-3$ & 3.97 & $2.1 \mathrm{e}-5$ & 5.00 & $2.7 \mathrm{e}-3$ & 4.52 & $3.6 \mathrm{e}-09$ \\
& 5 & $2.1 \mathrm{e}-4$ & 4.00 & $6.6 \mathrm{e}-7$ & 5.01 & $9.5 \mathrm{e}-5$ & 4.85 & $6.7 \mathrm{e}-09$ \\
\hline
\end{tabular}

Table 4.1: Errors for Kovasznay flow $(\nu=1)$ and pairs $\mathrm{RT}_{k} / \mathbb{Q}_{k}$.

value of the nonlinear iteration to this value. In all subsequent iteration steps, the residual and update vectors are set to zero in the corresponding components. The tangential component of the velocity field is prescribed in weak form, as with standard DG schemes.

We test our method with the analytical solution given in [19], namely

$$
\begin{aligned}
u_{1}(x, y) & =1-e^{\lambda x} \cos (2 \pi y) \\
u_{2}(x, y) & =\frac{\lambda}{2 \pi} e^{\lambda x} \sin (2 \pi y) \\
p(x, y) & =-\frac{1}{2} e^{2 \lambda x}+\bar{p}
\end{aligned}
$$

where

$$
\lambda=\frac{-8 \pi^{2}}{\nu^{-1}+\sqrt{\nu^{-2}+16 \pi^{2}}} .
$$

We impose this function as Dirichlet boundary condition on the boundary of $\Omega=$ $\left(-\frac{1}{2}, \frac{3}{2}\right) \times(0,2)$ and choose a viscosity $\nu=1$. Absolute errors and convergence rates for several pairs of $\mathrm{RT}_{k} / \mathbb{Q}_{k}$ polynomials are listed in Table 4.1. It exhibits clearly the expected convergence orders for error of the velocity in $H^{1}\left(\mathbb{T}_{h}\right)$ and $L^{2}(\Omega)$. The pressure errors in this table converge much faster than expected; a fact we cannot explain right now. 


\section{Concluding remarks}

In this note, we elaborated on remarks in [15] about DG methods for the NavierStokes (or for incompressible fluid flow) which can provide exactly incompressible velocity approximations and how this can be achieved essentially independently of the DG discretization of the viscous terms. Although we focused on the Raviart-Thomas pair of local spaces $R T_{k} / \mathbb{Q}_{k}$ on rectangles, our results apply to other pairs of elements originally designed for the discretization of the Poisson problem in mixed form.

\section{References}

[1] D. N. Arnold. An interior penalty finite element method with discontinuous elements. SIAM J. Numer. Anal., 19:742-760, 1982.

[2] D. N. Arnold, F. Brezzi, B. Cockburn, and L. D. Marini. Unified analysis of discontinuous Galerkin methods for elliptic problems. SIAM J. Numer. Anal., 39:1749-1779, 2002.

[3] G. A. Baker. Finite element methods for elliptic equations using nonconforming elements. Math. Comput., 31:45-59, 1977.

[4] F. Bassi and S. Rebay. A high-order accurate discontinuous finite element method for the numerical solution of the compressible Navier-Stokes equations. J. Comput. Phys., 131:267-279, 1997.

[5] F. Bassi, S. Rebay, G. Mariotti, S. Pedinotti, and M. Savini. A highorder accurate discontinuous finite element method for inviscid and viscous turbomachinery flows. In R. Decuypere and G. Dibelius, editors, 2nd European Conference on Turbomachinery Fluid Dynamics and Thermodynamics, pages 99-108, Antwerpen, Belgium, March 5-7 1997. Technologisch Instituut.

[6] P. Bastian and B. Rivière. Superconvergence and $H($ div) projection for discontinuous Galerkin methods. Internat. J. Numer. Methods Fluids, 42:1043-1057, 2003.

[7] C. E. Baumann and J. T. Oden. A discontinuous $h p$-finite element method for convection-diffusion problems. Comput. Methods Appl. Mech. Engrg., 175:311-341, 1999.

[8] F. Brezzi, G. Manzini, L. D. Marini, P. Pietra, and A. Russo. Discontinuous finite elements for diffusion problems. In in Atti Convegno in onore di F. Brioschi (Milan 1997), pages 197-217. Istituto Lombardo, Accademia di Scienze e Lettere, 1999.

[9] J. Carrero, B. Cockburn, and D. Schötzau. Hybridized, globally divergencefree LDG methods. Part I: The Stokes problem. Math. Comput., 75:533$563,2006$. 
[10] P. Castillo, B. Cockburn, I. Perugia, and D. Schötzau, An a priori error analysis of the local discontinuous Galerkin method for elliptic problems, SIAM J. Numer. Anal., 38 (2000), pp. 1676-1706.

[11] B. Cockburn and J. Gopalakrishnan. Incompressible finite elements via hybridization. Part I: The Stokes system in two space dimensions. SIAM J. Numer. Anal., 43:1627-1650, 2005.

[12] B. Cockburn and J. Gopalakrishnan. Incompressible finite elements via hybridization. Part II: The Stokes system in three space dimensions. SIAM J. Numer. Anal., 43:1651-1672, 2005.

[13] B. Cockburn, G. Kanschat, and D. Schötzau. Local discontinuous Galerkin methods for the Oseen equations. Math. Comput., 73:569-593, 2004.

[14] B. Cockburn, G. Kanschat, and D. Schötzau. The local discontinuous Galerkin methods for linear incompressible flow: A review. Computers and Fluids (Special Issue: Residual distribution schemes, discontinuous Galerkin schemes and Adaptation), 34:491-506, 2005.

[15] B. Cockburn, G. Kanschat, and D. Schötzau. A locally conservative LDG method for the incompressible Navier-Stokes equations. Math. Comput., 74:1067-1095, 2005.

[16] B. Cockburn, G. Kanschat, D. Schötzau, and C. Schwab. Local discontinuous Galerkin methods for the Stokes system. SIAM J. Numer. Anal., 40:319-343, 2002.

[17] B. Cockburn, D. Schötzau, and J. Wang. Discontinuous Galerkin methods for incompressible elastic materials. Comput. Methods Appl. Mech. Engrg. To appear.

[18] B. Cockburn and C.-W. Shu. The local discontinuous Galerkin method for time-dependent convection-diffusion systems. SIAM J. Numer. Anal., $35: 2440-2463,1998$.

[19] L. Kovasznay. Laminar flow behind a two-dimensional grid. Proc. Camb. Philos. Soc., 44:58-62, 1948.

[20] B. Rivière, M. F. Wheeler, and V. Girault. Improved energy estimates for interior penalty, constrained and discontinuous Galerkin methods for elliptic problems. Part I. Comput. Geosci., 3:337-360, 1999.

[21] D. Schötzau, C. Schwab, and A. Toselli. hp-DGFEM for incompressible flows. SIAM J. Numer. Anal., 40:2171-2194, 2003. 